\title{
Propriedades mecânicas do Concreto de Ultra Alto Desempenho Reforçado por Fibras (UHPFRC): uma revisão de literatura
}

\author{
Mechanical behaviorof Ultra High Fibers Reinforced \\ Concrete (UHPFRC): a state of art
}

Giovanna Zaramella ${ }^{1}$, Silvana Ortolan Hardt ${ }^{1}$, Willian Lucas Parabocz ${ }^{1}$, Carlos Francisco Pecapedra $^{1}$, Gustavo de Miranda Saleme Gidrão ${ }^{*}$, Rúbia Mara Bosse¹, Rodrigo Scoczynski

Ribeiro ${ }^{1}$, Dyorgge Alves Silva ${ }^{1}$

\begin{abstract}
RESUMO
O presente artigo endereça uma breve revisão de literatura sobre comportamento do Concreto de Ultra Alto Desempenho Reforçado por Fibras (UHPFRC). É apresentada a abordagem sobre a sua durabilidade, suas propriedades mecânicas e sua resistência, quando exposto ao ataque de ácidos. O UHPFRC vem se destacando como um material emergente, onde apresenta alta resistência em relação ao concreto convencional (maior ou igual a $100 \mathrm{MPa}$ na compressão). Sob a ótica de durabilidade, são apresentadas pesquisas as quais propiciam uma análise comparativa em relação a utilização do concreto convencional, do Concreto de Ultra Alto Desempenho (UHPC) e do UHPFRC, bem como análises comparativas entre as diferentes abordagens do próprio UHPFRC e estudos acerca de ataques ácidos. Este artigo avança na compilação de trabalhos sobre o UHPC e UHPFRC.
\end{abstract}

Palavras-chave: UHPFRC; Fibras; Propriedades mecânicas; Ataque ácido

\begin{abstract}
This paper presents a study about the behavior of Ultra High Performance Fiber Reinforced Concrete (UHPFRC). It presents a brief overview of its durability, its mechanical properties and its resistance when exposed to acid attack. The UHPFRC has stood out as a more resistant material than the conventional concrete, (more or same as $100 \mathrm{MPa}$ on compression resistence). Looking on durability, surveys are presented which provide a comparative analysis between the conventional concrete, the ultra high performance concrete (UHPC) and the UHPFRC, as well as comparative analyses between the different approaches of the own UHPFRC and studies about acid attacks. This article has advanced on a compilation of works about UHPC and UHPFRC.
\end{abstract}

Keywords: UHPFRC; Fibers; Mechanical properties; Acid attack

\footnotetext{
${ }^{1}$ UTFPR - Universidade Tecnológica Federal do Paraná 1. *E-mail: gidrao@utfpr.edu.br
} 


\section{INTRODUÇÃO}

Em 1993, o trabalho de Richard e Cheyrezy foi precursor ao elevar as características mecânicas de misturas cimentícias a patamares até então nunca vistos. Assim, foi desenvolvido o concreto de pós reativos (CPR), por meio de utilização de partículas mais finas, adições minerais e tratamento térmico (CHEYREZY; RICHARD, 1994; RICHARD; CHEYREZY, 1995). Esses materiais se destacaram por sua grande durabilidade e alta resistência, características observadas devido à seleção rigorosa de materiais, projeto de empacotamento de partículas e processo de mistura padronizado (ALI, 2013; HABEL; GAUVREAU, 2008; SPASOJEVIĆ, 2008). Assim, o CPR apresentava resistência à compressão na ordem de 150-200 MPa com cura de autoclave (CHEYREZY; RICHARD, 1994; RICHARD et al., 1995) e porosidade inferior a 4\% (ZDEB, 2013). Portanto, devido a suas características superiores de baixa porosidade e consideráveis durabilidade e resistência, o material tornou-se proeminente na utilização em instalações de armazenamento de resíduos industriais e nucleares (YAZICI et al., 2015).

Com notórios avanços tecnológicos dos superplastificantes e adições minerais, permitiu-se a produção de concretos com altas proporções de finos, perfeitamente empacotados e com baixas relações água/aglutinante (ZDEB, 2013; FEHLING et al., 2014). Segundo Shi et al. (2015), o termo de "Concreto de Ultra Alto desempenho" (CUAD ou UHPC - Ultra-High-Performance Concrete) aparece no ano de 1994, quando De Larrard e Sedran (1994) otimizam uma mistura de CPR por meio do empacotamento de grãos, tendo denominado esse novo traço de UHPC. Nesse sentido, é notório que a mistura de CPR é o grande precursor do UHPC (DE LARRARD; SEDRAN, 1994; AÏTCIN, 2007). Dessa forma, como requisitos básicos, o UHPC deverá apresentar (a) propriedades mecânicas elevadas no longo prazo, (b) baixa permeabilidade e (c) longa vida em ambientes adversos (ZDEB, 2013; HANNA; MORCOUS; TADROS, 2014).

É notório que atualmente existem várias definições de UHPC na literatura, mas é bem aceito que se trata de uma mistura cimentícia com relação água-cimento $(\mathrm{a} / \mathrm{c})$ de aproximadamente 0,2 e resistência à compressão aos 28 dias de no mínimo $100 \mathrm{MPa}$ (HUNG; LEE; CHAN, 2019; YAN et al., 2018), resistência à tração de 5 MPa (AFGC, 2013; HABEL et al., 2006; RUSSEL; GRAYBEAL, 2013; TUAN et al., 2011; WILLE et al., 2011; WU et al., 2016), sendo ainda esses valores de 5 a 16 vezes maiores que 
aqueles observados em concreto convencional (WANG et al., 2015). No entanto, com o aumento severo de resistência e a diminuição das imperfeições da microestrutura, o material tende a romper de forma frágil e brusca. Para contornar essa limitação, a fragilidade é geralmente compensada pela adição de fibras, o que transforma o UHPC em UHPFRC - Ultra High-Performance Fiber-Reinforced Concrete (FEHLING et al., 2014).

Como estimativa inicial sobre as propriedades mecânicas e de durabilidade de uma mistura de UHPC/UHPFRC com cura a vapor, Cavill et al. (2006) elaboraram as Tabelas 1 e 2 .

Tabela 1 - Propriedades mecânicas usuais do UHPFRC

\begin{tabular}{cc}
\hline Propriedade & Valor \\
\hline Fluidez - Flow table & $190-200 \mathrm{~mm}$ após 20 golpes \\
Resistência à compressão com cura & $150 \mathrm{MPa}$ \\
térmica \\
Resistência à tração sob flexão \\
Primeira fissura em flexão \\
Módulo de elasticidade \\
Densidade & $24 \mathrm{MPa}$ \\
& $20 \mathrm{MPa}$ \\
Retração & $47 \mathrm{GPa}$ \\
& $2.450 \mathrm{~kg} / \mathrm{m}^{3}$ \\
& $<500$ micros depois de 50 dias \\
& em cura úmida e 0 em cura com \\
\hline
\end{tabular}

Fonte: Cavill, Rebentrost e Perry (2006)

Tabela 2 - Propriedades de durabilidade do UHPC

\begin{tabular}{cc}
\hline Propriedade & Valor \\
\hline Porosidade total & $2-6 \%$ \\
& \\
Microporosidade & $<1 \%$ \\
\hline
\end{tabular}




\begin{tabular}{cc}
\hline Absorção de água & $<0,2 \mathrm{~kg} / \mathrm{m}^{2}$ \\
Difusão de íons $\mathrm{Cl}^{-}$ & $0,02 \mathrm{E}-12 \mathrm{~m}^{2} / \mathrm{s}$ \\
Resistência elétrica (s/ fibra) & $1,13 \mathrm{E} 3 \mathrm{kwcm}$ \\
Resistência elétrica (c/ fibra) & $137 \mathrm{kwcm}$ \\
Coeficiente de abrasão & 1,3 \\
\hline
\end{tabular}

Fonte: Cavill, Rebentrost e Perry (2006)

Devido às propriedades mecânicas e de durabilidade, o UHPFRC tornou-se em pouco tempo um material emergente, com várias aplicações na indústria (AHLBORN; PUESE; MISSON, 2008; REBENTROST; WIGHT, 2008; SALEEM, 2011), fornecendo soluções econômicas, sustentáveis, duráveis e inovadoras em áreas em que o concreto normal luta para encontrar uma solução viável.

Portanto e de acordo com o contexto supracitado, o presente artigo tem por objetivo apresentar uma revisão bibliográfica sobre as principais propriedades mecânicas do UHPFRC sob condições normais e sob ataque de ácidos. Portanto, as próximas seções serão focadas em (i) apresentar os aspectos à respeito do traço do UHPFRC, (ii) quantificar as principais propriedades mecânicas observadas na literatura, (iii) descrever alguns experimentos condicionados a durabilidade do material, e (iv) exemplificar algumas das aplicações do UHPFRC no munndo. Este artigo é parte de um projeto de pesquisa desenvolvido na UTFPR Guarapuava, que tem como objetivo contribuir com o desenvolvimento do dimensionamento, simulação e análise do material.

\section{METODOLOGIA}

No mês de setembro de 2021 foi realizada uma revisão bibliográfica utilizando a ferramenta do google scholar, focando nas palavras chave de UHPFRC, UHPC, durabilidade e durability. As principais propriedades em corpos de prova, tais como resistência à compressão, resistência à tração e porosidade foram obtidas para os UHPFRCs, em situação de pH normal e sob ataque de ácidos. 


\section{RESULTADOS OBTIDOS DA LITERATURA E DISCUSSÕES}

\section{Dosagem: Estratégias para obtenção de um traço de UHPC e UHPFRC}

Quando comparado aos concretos usuais, o traço de UHPFRC apresenta baixa proporção de água-cimento e alta dosagem de fibras, com o objetivo de oferecer um melhor desempenho em relação a sua resistência e ductilidade. Dessa maneira, é imprescindível conhecer melhor suas propriedades e apresentar seu desempenho em relação a outros concretos (BUTTIGNOL; SOUSA; BITTENCOURT, 2017).

O UHPFRC é feito através de uma mistura de Cimento Portland, sílica ativa, pó de quartzo, areia de sílica fina, água, superplastificantes e microfibras orgânicas ou de aço. Nesse caso a relação água/cimento é menor em comparação ao concreto tradicional e a trabalhabilidade desse material no estado fresco também é superior (BUTTIGNOL; SOUSA; BITTENCOURT, 2017).

Também podem ser acrescidos alguns materiais recicláveis e de resíduos industriais, justamente pensando em eficiência energética e com intuito de testar novas maneiras de construir de forma mais eficiente e sustentável (YU; SPIEZ; BROUWERS, 2015). Desta forma, o UHPFRC, pode ser apresentado como alternativa viável para obtenção de eficiência tanto ecológica, quanto tecnológica, uma vez que o material permite reduções do consumo de cimento.

Rejeitos e subprodutos industriais, tais como a escória de alto forno granulada e pulverizada, cinzas volantes pulverizadas, metacaulim, pó de calcário, cinzas de casca de arroz e pó de escória de aço podem ser utilizados com sucesso como adição mineral no UHPC, ajudando a promover benefícios tais como a reação pozolâmica, preenchimento de poros e restrição da liberação do calor de hidratação (PENG; HU; DING, 2010; SHI et al., 2015; SHI; MATSUI; FENG, 2002; TAFRAOUI et al., 2009; TUAN et al., 2011; VAN TUAN et al., 2011; WANG et al., 2012; YAZICI et al., 2008, 2010; ZHU; GIBBS, 2005). Tais subprodutos permitem que o traço de UHPFRC apresente sustentabilidade necessária. De forma similar, nanopartículas como a nano-sílica (nano-SiO 2 ), o nano- 
calcário (nano-CaCO 3 ), óxido de nano-titânio $\left(\mathrm{Nano}-\mathrm{TiO}_{2}\right)$ e o nano-ferro $\left(\mathrm{Nano}-\mathrm{Fe}_{2} \mathrm{O}_{3}\right)$ também podem diminuir a porosidade e também melhorar o comportamento do material (SANCHEZ; SOBOLEV, 2010).

Toledo Filho et al. (2012) desenvolveram um UHPFRC sustentável baseado em cimento de escória de alto forno, sílica, microfibras de wollastonita, fibras de aço e superplastificante. Mais uma vez, observou-se rápido desenvolvimento da resistência a compressão, sendo obtido para o terceiro dia um valor de $\mathrm{f}_{\mathrm{c}}$ igual à $75 \mathrm{MPa}$, seguido por $111 \mathrm{MPa}, 162 \mathrm{MPa}$ e $180 \mathrm{MPa}$ para as idades subsequentes de 7, 28 e 180 dias.

Cabe ressaltar que os resultados de $\mathrm{f}_{\mathrm{c}}$ pela maturidade, como os de Corinaldesi e Moriconi (2012), Habel et al. (2006) e Toledo Filho et al. (2012), demonstram o potencial de aplicabilidade do UHPFRC para indústria de pré moldados, uma vez que o rápido desenvolvimento de $f_{c}$ em idades precoces pode acelerar o processo de fabricação e execução de estruturas pré fabricadas.

Embora tenham poucos estudos em relação ao traço ideal, muitas pesquisas tais como Torregrosa (2013), Balestra, Ozelame e Savaris (2020) e Rompa e Gidrão (2020) indicam alguns traços usuais que são utilizados de forma experimental com o intuito de estudar propriedades como a resistência e rigidez do UHPFRC.

De acordo com Torregrosa (2013), algumas estratégias básicas para obtenção de uma mistura de UHPFRC, podem ser resumidas conforme a Fig. 1. Tais estratégias são utilizadas conjuntamente com o intuito de otimizar os traços, reduzindo a porosidade e aumentando a resistência. De fato, o primeiro passo para a dosagem de um traço de UHPFRC é a seleção criteriosa de materiais, com grande controle tecnológico e de qualidade. Por exemplo, o cimento utilizado não pode estar na condição empedrada, bem como a água deve ser potável e os agregados secos e com adequada distribuição granulométrica. Uma alternativa viável é a utilização de areias industriais, como no estudo de Rompa e Gidrão (2020). A eliminação do agregado graúdo diminui na mistura a zona de transição e aumenta a homogeneidade da mistura, melhorando a resistência e diminuindo a porosidade. Aditivos superplastificantes permitem a diminuição da relação água-cimento e aumento da trabalhabilidade, no entanto podem causar retardamento de pega. Com o aumento de trabalhabilidade é possível a utilização de fibras metálicas, que aumentam a resistência à tração e ductilidade do material, permitindo rupturas com altas energias de deformação. Adições minerais como a sílica ativa e o pó de quartzo permitem 
o desenvolvimento de reações pós reativas, transformando o hidróxido de cálcio da zona de transição em CSH tardio (ROMPA; GIDRÃO, 2020). Segundo Zhang e Li (2013) no UHPFRC, a utilização da sílica diminui a porosidade do concreto no momento de hidratação inicial, o que resulta em menor absorção de água no estado endurecido. Sendo assim, a proporção de sílica utilizada influencia na durabilidade do concreto.

Figura 1 - Princípios do UHPFRC

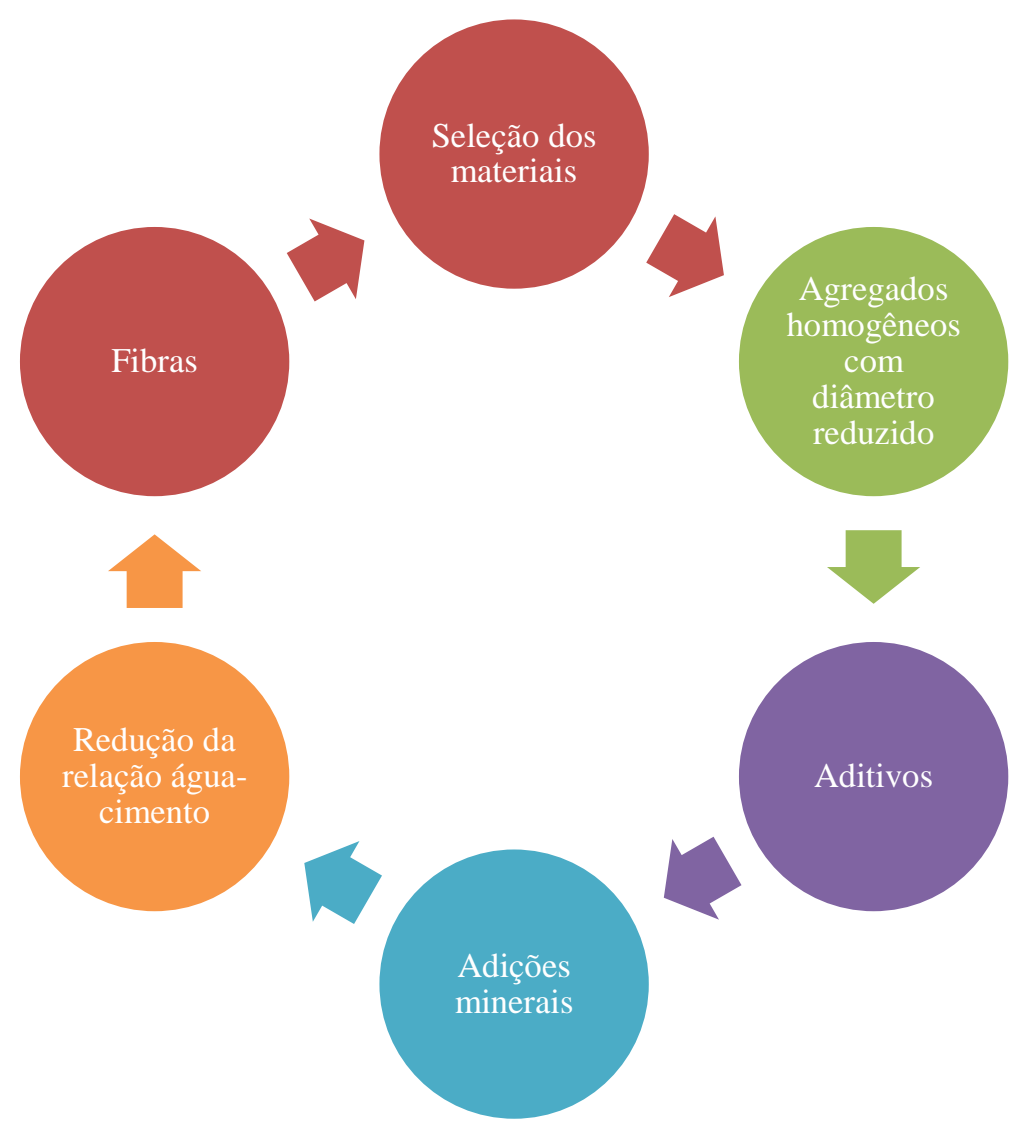

Fonte: Adaptado de Torregrosa (2013)

Após a aplicação das estratégias da Fig. 1, inicia-se o processo de mistura (ROMPA; GIDRÃO, 2020) seguindo algumas etapas: os materiais secos são adicionados e misturados durante 5 minutos, na etapa seguinte são acrescentados o superplastificante e a água, e novamente a mistura é submetida a homogeneização por aproximadamente 10 minutos. Em seguida, são adicionadas as fibras e novamente é efetuada a mistura durante aproximadamente 5 minutos. Posteriormente são realizados testes de espalhamento e, logo após, são moldados corpos de prova cilíndricos para efetuar os testes de compressão e verificação do módulo de elasticidade do material. 
Figura 2 - Procedimento de fabricação do UHPFRC/UHPC

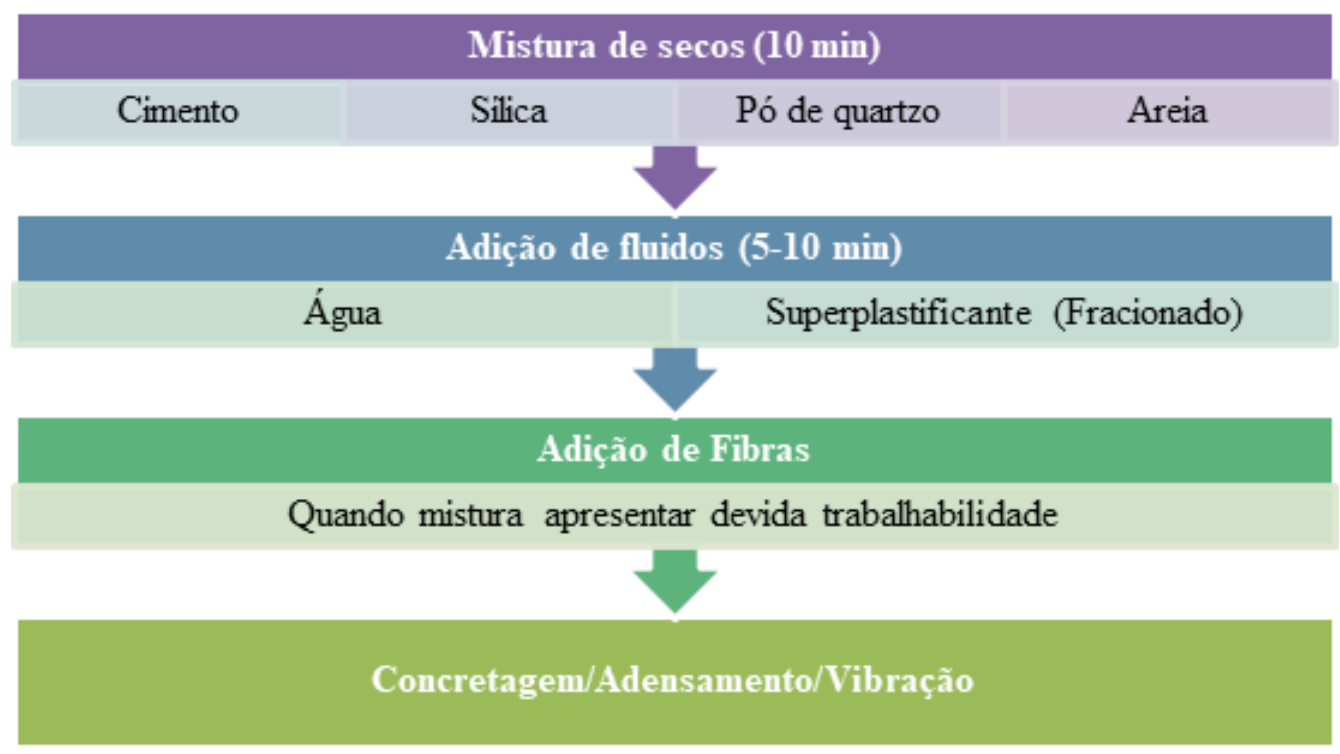

Fonte: Adaptado de YU et. al. (2014)

Uma das características principais do concreto de alto desempenho é a ausência de agregados graúdos, a zona de transição também é menor e mais resistente (BALESTRA; OZELAME; SAVARIS, 2020), ou seja, no caso do UHPFRC não são observadas microfissurações prévias tais como nos concretos tradicionais, implicando em controle de fissuração e aumento da resistência.

A trabalhabilidade do concreto de alto desempenho é um fator que determina essa orientação das fibras, e pode ser considerada característica essencial para implicar em qualidade no processo construtivo. (BOULEKBACHE; HAMRAT; CHEMROUK, AMZIANE, 2010).

Nos estudos realizados por Máca et al. (2013) foram realizados testes com intuito de comparar as propriedades mecânicas de traços de UHPFRC, NSC (Normal Strength Concrete), FRC (Fiber Reinforced Concrete) e o HPC (High Performance Concrete), com as composições em kg/m³ apresentadas na Tabela 3.

Os traços de NSC e o FRC foram desenvolvidos com o objetivo de se atingir 30 MPa de resistência à compressão aos 28 dias (fck), utilizando o consumo de $300 \mathrm{~kg} / \mathrm{m}^{3}$ 
de cimento e aditivo redutor de água para melhorar a trabalhabilidade. Já para o HPC e o UHPFRC foi utilizada uma grande quantidade de cimento e também fibras em $2 \%$ do volume. Os traços são apresentados na Tabela 3:

Tabela 3 - Composição dos concretos em kg/m³

\begin{tabular}{|c|c|c|c|c|}
\hline Componente & NSC & FRC & HPC & UHPFRC \\
\hline Cimento, CEM $142,5 \mathrm{R}$ & 320 & 370 & - & - \\
\hline Cimento, CEM 1 52,5 R & - & - & 800 & 800 \\
\hline Água & 155 & 175 & 176 & 176 \\
\hline Redutor de água & 1,45 & 3,5 & - & - \\
\hline Redutor de água de alta variação & - & - & 40 & 40 \\
\hline Agregado $0 / 4 \mathrm{~mm}$ & 850 & 1130 & - & - \\
\hline Agregado $4 / 8 \mathrm{~mm}$ & 800 & 750 & - & - \\
\hline Areia fina $0.1 / 0.6 \mathrm{~mm}$ & - & - & 336 & 336 \\
\hline Areia fina $0.3 / 0.8 \mathrm{~mm}$ & - & - & 800 & 640 \\
\hline Microsílica & - & - & 200 & 200 \\
\hline Pó de vidro & - & - & 200 & 200 \\
\hline Fibras $(13 \mathrm{x} 0,15 \mathrm{~mm})$ & - & - & - & 160 \\
\hline Fibras $(32 \times 0,55 \mathrm{~mm})$ & - & 50 & - & - \\
\hline
\end{tabular}

Fonte: Máca, Sovják e Vavřiník (2013)

Segundo o gráfico da Fig. 3, nota-se por meio das porcentagens de materiais, as estratégias de obtenção do UHPFRC descritas acima (vide Fig.2). Assim, uma mistura de UHPFRC se diferencia das outras misturas de concreto por ter fibras de menor dimensão, ausência de agregado graúdo, utilização de pós reativos e outros ultrafinos (e.g., pó de vidro) e redutores de água do tipo superplastificante. Os resultados destes traços podem ser contemplados na Tabela 4 que segue no presente trabalho. 
Figura 3 - Porcentagens em materiais dos traços de NSC, HPC, FRC e UHPFRC

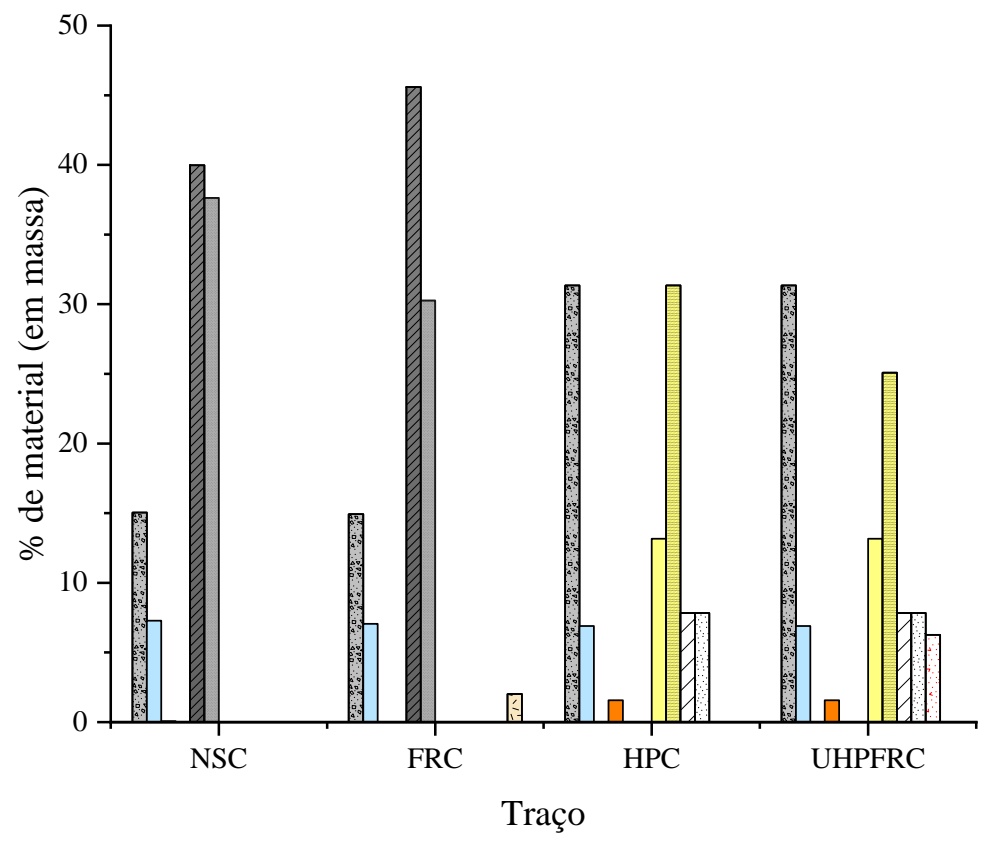

\begin{tabular}{|c|c|}
\hline 28 & Cimento \\
\hline & Água \\
\hline & Redutor de água \\
\hline & Redutor de água de alta variação (Superplastificante) \\
\hline & Agregado $0 / 4 \mathrm{~mm}$ \\
\hline & Agregado $4 / 8 \mathrm{~mm}$ \\
\hline & Areia fina $0.1 / 0.6 \mathrm{~mm}$ \\
\hline & Areia fina $0.3 / 0.8 \mathrm{~mm}$ \\
\hline EA & Microsílica \\
\hline & Pó de vidro \\
\hline & Fibras $(13 \times 0,15 \mathrm{~mm})$ \\
\hline & Fibras $(32 \times 0,55 \mathrm{~mm})$ \\
\hline
\end{tabular}

Fonte: Adaptado de Máca, Sovják e Vavřiník (2013)

Propriedades mecânicas do UHPFRC: valores usuais da literature 
$\mathrm{Na}$ bibliografia, extensivas pesquisas são desenvolvidas, onde experimentos comprovam a eficácia mecânica do UHPFRC quando comparado a outros tipos de concretos. Assim, propriedades como a resistência mecânica à compressão, tensão e flexão e módulo de elasticidade. Vale ressaltar, contudo, que muitas variáveis interferem na obtenção de certos resultados, como a quantidade, a geometria e a orientação das fibras, o procedimento de cura e de mistura, o tamanho dos corpos de prova utilizados, entre outros fatores (SHAIKH et. al., 2020), abrindo campo para futuros estudos.

Na Tabela 4, é apresentada uma relação comparativa das propriedades mecânicas observadas nos estudos de Máca et al (2013). Com seus experimentos foi possível observar que o UHPFRC consegue atingir propriedades mecânicas melhores que os demais tipos de concreto, utilizando técnicas padrão de mistura e de cura. As maiores desvantagens observadas no UHPFRC, segundo os autores, é a alta viscosidade, altos custos e altas exigências de controle de qualidade nos constituintes. De fato, a alta viscosidade pode gerar segregação de materiais, bem como o retardamento da pega. Já o custo está atrelado principalmente ao valor agregado dos insumos e ao alto gasto de cimento Portland nas misturas.

Tabela 4 - Propriedades Mecânicas dos concretos estudados por Máca et al.

\begin{tabular}{ccccc}
\hline Propriedade [Unidade] & NSC & FRC & HPC & UHPFRC \\
\hline Resistência à compressão [MPa] & 42,8 & 37,4 & 132,4 & 151,7 \\
Módulo de Elasticidade [GPa] & 35,5 & 29,8 & 41,1 & 47,5 \\
$\quad$ Resistência à flexão [MPa] & 6,2 & 7,1 & 13,9 & 29,7 \\
$\quad \begin{array}{l}\text { Ponto médio de deflexão `- flexão de 3 } \\
\quad \text { pontos [mm] }\end{array}$ & 0,16 & 0,29 & 0,1 & 1,36 \\
$\quad$ Resistência à tração direta [MPa] & - & - & 6,6 & 10,3 \\
Energia de fratura efetiva [J/m²] & 165 & 3955 & 347 & 19847 \\
\hline
\end{tabular}

Fonte: Máca, Sovják e Vavřiník (2013)

Mesmo com as desvantagens supracitadas, a Fig. 4 representa a sensível melhoria das propriedades de resistência à compressão (fc), módulo de elasticidade secante (Ecs) e Módulo de ruptura flexional (MRF) em comparação aos concretos NSC, FRC e HPC 
apresentados por Máca, Sovják e Vavřiník (2013). Nota-se um aumento de 350 \% em relação ao valor de resistência média, 33 \% para o módulo de elasticidade e 479 \% para a resistência à flexão. Com este resultado, pode-se verificar que os estados limite de serviço são mais críticos para o UHPFRC, uma vez que a resistência de flexão e de compressão do material tem sensível melhora em relação à rigidez.

Figura 4 - Propriedades do UHPFRC

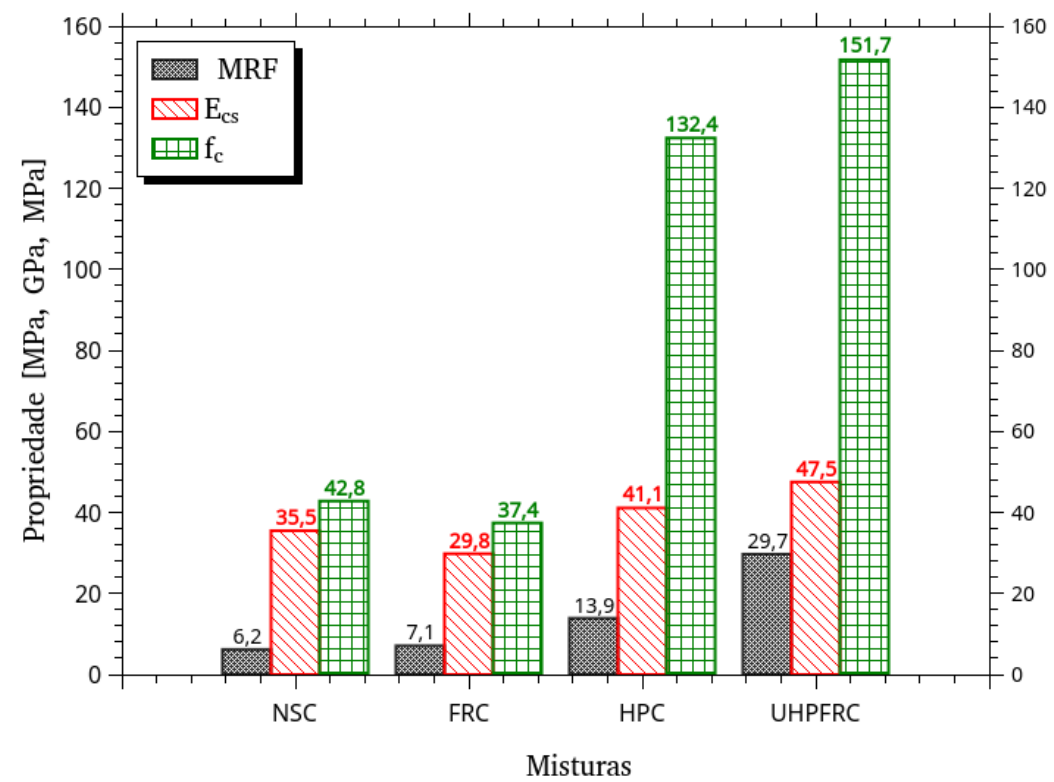

Fonte: Adaptado de Máca, Sovják e Vavřiník (2013)

Uma outra frente de estudo é na utilização de outros tipos de fibras. Por exemplo, no trabalho desenvolvido por Mohammed et al (2020), optou-se pela substituição da fibra metálica pelas micro fibras de vidro MGF (micro glass fiber). Como agregado fino foi usado o quartzo comercial em tamanhos de diferentes frações de 1,2 - 2,5mm, 0,6 - 1,2mm e $0,0-0,4 \mathrm{~mm}$ com peso específico de $2,65 \mathrm{~g} / \mathrm{cm}^{3}$. Para melhorar a trabalhabilidade foi utilizado um superplastificante policarboxilato. As fibras usadas do tipo MGF (micro glass fiber) com $13 \mathrm{~mm}$ de comprimento e $18 \mu \mathrm{m}$ de diâmetro. Os corpos de prova foram moldados com as dimensões citadas: $70 \times 70$ × $280 \mathrm{~mm}$, para determinar as propriedades mecânicas. Para esse estudo foi analisado como a relação do fator água cimento afeta no resultado final obtido no concreto, adotando valores de 0,12 e 0,14 , bem como para diferentes porcentagens de volume de fibra. Foi percebido que quanto menor o fator a/c, melhor foram os resultados quanto às propriedades mecânicas analisadas. Dentre os 
resultados destacam-se os valores máximos obtidos: a resistência à compressão de 160 MPa e módulo de elasticidade máximo de aproximadamente 43,2GPa, sendo os dois para a amostra de 1,5\% de volume de fibra e 0,12 de fator a/c. Já o maior módulo de ruptura encontrado foi de aproximadamente $17,8 \mathrm{MPa}$ e a máxima energia de ruptura foi de aproximadamente $540 \mathrm{~N} / \mathrm{m}$, sendo esses para as amostras de $3 \%$ de volume de fibra e fator a/c de 0,12 .

No estudo realizado por Hannawi et al. (2016), foi utilizado o Cimento Portland CEM I 52,5 com fíler de calcário e fíler de silício. Como agregado foi adicionado areia silicosa, com densidade aparente de $1730 \mathrm{~kg} / \mathrm{m}^{3}$, peso específico de 2,65 e fator água cimento de 0,27. Para esse experimento foi utilizado como variável o tipo de fibra, diferindo pela sua natureza (aço, mineral e sintético), diferentes dimensões (macro e microscopicamente) e pelas propriedades mecânicas. Sendo adicionado $1 \%$ de volume de fibra em todas as amostras. Os valores máximos obtidos nos experimentos foram 173,2 MPa de resistência à compressão, módulo de elasticidade de 51,8 GPa e limite de ruptura de 166,3 $\mathrm{MPa}$, sendo a amostra de fibras de aço a que apresentou esse melhor desempenho.

No entanto, a diminuição da relação água-cimento nem sempre conduz a misturas com maior resistência. Um estudo importante conduzido por Corinaldesi e Moriconi (2012), avaliou a influência do fator a/c nas misturas de UHPFRC. As amostras foram curadas a temperatura ambiente $\left(20^{\circ} \mathrm{C}+100 \%\right.$ U.R.) com relações água-cimento variáveis de $0,20-0,32$. Os resultados demonstram que o aumento progressivo da relação $\mathrm{a} / \mathrm{c}$ nem sempre conduziu a matrizes de UHPC menos resistentes, uma vez que para o valor intermediário de $\mathrm{a} / \mathrm{c}=0,24$ observa-se o melhor resultado de $\mathrm{f}_{\mathrm{c}}$, diferentemente do que é esperado para casos de concretos convencionais. Esta observação experimental é explicada pelo fato de que as propriedades da mistura fresca tais como coesão e trabalhabilidade passam a influenciar severamente as propriedades mecânicas do UHPC/UHPFRC. 
Figura 5 - Influência da maturidade e relação água-cimento nos traços de UHPFRC

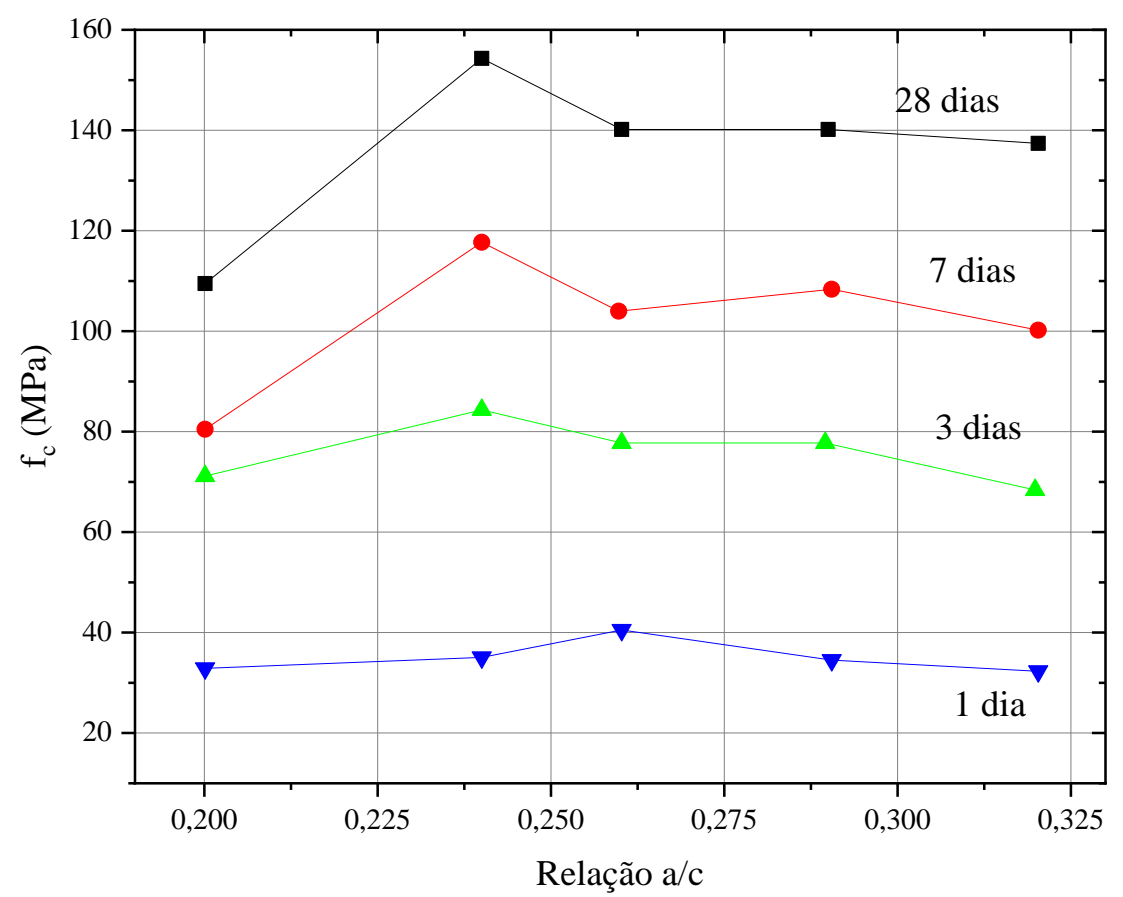

Fonte: Corinaldesi e Moriconi (2012)

$\mathrm{Na}$ abordagem feita por Yoo et al. (2013), foram analisadas diferentes propriedades mecânicas do UHPFRC variando o volume de fibras $(1 \%, 2 \%, 3 \%$ e 4\%) de $13 \mathrm{~mm}$ de comprimento e $0,2 \mathrm{~mm}$ de diâmetro, para uma matriz de argamassa idêntica. Foi utilizado um fator a/c de 0,2 e um superplastificante de policarboxilato, as amostras passaram por processo de cura a $\left(90 \pm 2^{\circ} \mathrm{C}\right)$. Os melhores valores obtidos nos experimentos foram de 207,2 MPa de resistência à compressão e 52,7 GPa de módulo de elasticidade, os dois valores foram obtidos com a amostra de 3\% de volume de fibra. Já para a resistência à flexão, o melhor desempenho foi de 50,81 MPa advindo da amostra de $4 \%$ de volume de fibra. 
Já o estudo conduzido por Rompa e Gidrão (2020) analisou a resistência à compressão e o módulo de elasticidade do UHPFRC. Os materiais utilizados foram cimento, areia, sílica, fibras (1,165\%), água e superplastificante (9\%). Foram moldados os corpos de prova cilíndricos $5 \mathrm{~cm}$ de diâmetro x $10 \mathrm{~cm}$ de comprimento, obtendo um valor de $112 \mathrm{MPa}$ de resistência à compressão e 40 GPa de módulo de elasticidade.

$\mathrm{Na}$ tabela 5 podem ser observados em resumo os valores encontrados para o módulo de elasticidade e resistência à compressão dos artigos mencionados. Nota-se a grande variabilidade de resistência e rigidez entre os concretos.

Tabela 5 - Resultado de estudos de caso do UHPFRC

\begin{tabular}{c|c|c}
\hline Autores & Resistência à compressão (MPa) & $\begin{array}{c}\text { Módulo de elasticidade } \\
\text { (GPa) }\end{array}$ \\
\hline Mohammed et al (2020) & 160 & 43,2 \\
Hannawi et al (2016) & 173,2 & 51,8 \\
Yoo, Lee e Yoon (2013) & 207,2 & 52,7 \\
Rompa e Gidrão (2020) & 112 & 40 \\
Corinaldesi e Moriconi (2012) & 159 & 52.7 \\
Máximo & 173,2 & 40 \\
Mínimo & 112 & 46.9 \\
\hline
\end{tabular}

Fonte: Mohammed et al (2020); Hannawi et al (2016); Yoo, Lee e Yoon (2013); Rompa e Gidrão (2020); Corinaldesi e Moriconi (2012) 


\section{DURABILIDADE}

Outro ponto relevante sobre o estudo do UHPFRC é em relação à sua durabilidade a ataques ácidos, considerando a sua possibilidade de uso em estruturas em contato direto com estes materiais corrosivos.

No experimento feito por Guzzo (2018), foram comparadas amostras do concreto tradicional e amostras de UHPFRC (a fibra utilizada foi a de polipropileno), onde foram moldados os corpos de prova cilíndricos nas dimensões 10 x $20 \mathrm{~cm}$ e corpos de prova prismáticos de 10 x $10 \times 20 \mathrm{~cm}$, respectivamente. Ambas as amostras foram desmoldadas após três dias de cura, na sequência foram imersos em solução de cloreto de sódio 5\% durante 24 meses. A profundidade do ácido foi determinada após a aspersão de solução de nitrato de prata. O UHPFRC apresentou uma penetração de $1,4 \mathrm{~mm}$, enquanto o concreto convencional apresentou penetração ao ácido de 4,8 mm. Outra característica apresentada no concreto tradicional o cloreto impregnou toda a sua estrutural. Já para o UHPFRC houve penetração superficial do sulfato não danificando sua estrutura interna. De fato, Fernandes (2011) destaca que a baixa permeabilidade do UHPFRC garante uma maior resistência e durabilidade em relação ao ataque de ácidos, o que permite o contato desse material em meios agressivos, o material também pode ser utilizado como revestimento de pilares, para reparo de estruturas, barreiras protetoras, em pontes rodoviárias, entre outras aplicações.

O alto desempenho das propriedades mecânicas em ambientes adversos é garantido pela alta durabilidade. Tal propriedade é atingida pela microestrutura completamente densa e praticamente impermeável, o que faz com que o UHPC apresente alta resistência aos agentes agressivos. (RESPLENDINO, 2004) observa que a alta durabilidade do UHPC/UHPFRC é explicada pela comparação de seus índices de durabilidade com àqueles encontrados em concretos convencionais e de alta resistência, conforme a Erro! Fonte de referência não encontrada.6. Portanto, a porosidade praticamente nula garante vida útil muito mais longa quando comparado aos concretos usuais (GRAYBEAL; TANESI, 2008; PORTENEUVE; KORB; PETIT, 2001; TOLEDO FILHO et al., 2012), evitando à entrada de agentes agressivos. 
Tabela 6 - Índices de durabilidade do UHPC/UHPFRC

\begin{tabular}{cccc}
\hline Índice & Convencional & Alta resistência & UHPC/UHPFRC \\
\hline Índice de vazios [\%] & $14-20$ & $10-13$ & $1,5-5$ \\
$\begin{array}{c}\text { Permeabilidade ao } \\
\text { Oxigênio [m²] }\end{array}$ & $10^{-16}$ & $10^{-17}$ & $<10^{-19}$ \\
Difusão ao íon $\mathrm{Cl}^{-}$ & $2.10^{-11}$ & $2.10^{-12}$ & $2.10^{-14}$ \\
Teor de portlandite & & 0 \\
$\left(\mathrm{~kg} / \mathrm{m}^{3}\right)$ & 76 & 86 & 0 \\
\hline
\end{tabular}

Fonte: Resplendino (2004)

Um caso notável de durabilidade do UHPFRC é descrito por (GRAYBEAL; TANESI, 2008), onde demonstraram a aplicação bem sucedida do material em pavimentos rodoviários submetidos a alta ciclagem mecânica e térmica. Após alguns meses de vida útil do elemento solicitado sob tráfego intenso e mudanças climáticas, observou-se que o UHPFRC com $\mathrm{V}_{\mathrm{f}}=3 \%$ de fibras metálicas apresentou considerável robustez nas resistências à abrasão, fadiga, impacto, ataques químicos e ciclos de gelodegelo. Já Porteneuve, Korb e Petit (2001) avaliaram a microestrutura de amostras lixiviadas com vazão constante de $7 \mathrm{ml} / \mathrm{h}$ durante 1 ano. Após todo período de teste, por meio de microscopia eletrônica constatou-se não haver nenhuma mudança significativa na microestrutura do material e, além disso, surpreendentemente a lixiviação teve efeito positivo, pois a água potencializou a produção do C-S-H e a reação pozolânica.

Efetuando uma análise probabilística de confiabilidade, Toledo Filho et al. (2012) demonstram que, para o mesmo desempenho de durabilidade, o cobrimento de armaduras requerido pelo UHPFRC pode ser até 10 vezes menor quando comparado aos concretos convencionais de classe C40. Ademais, ainda que o cobrimento do UHPFRC seja de apenas $3 \mathrm{~mm}$ contra $35 \mathrm{~mm}$ de $\mathrm{C} 40$, analisando a fig. 6, pode-se notar que o comportamento da probabilidade de falha por durabilidade do UHPFRC em função do tempo é superior exclusivamente pelo fato de se manter praticamente nulo até os 50 anos de serviço.

Portanto, qualifica-se o UHPFRC como uma alternativa econômica, sustentável e adequada para proteger as armaduras imersas no concreto, sobretudo para situações em 
que a falha por durabilidade é crítica, como por exemplo as usinas nucleares, estruturas marinhas, tabuleiros rodoviários, etc.

Figura 6 - Probabilidade de falha de durabilidade para o UHPFRC

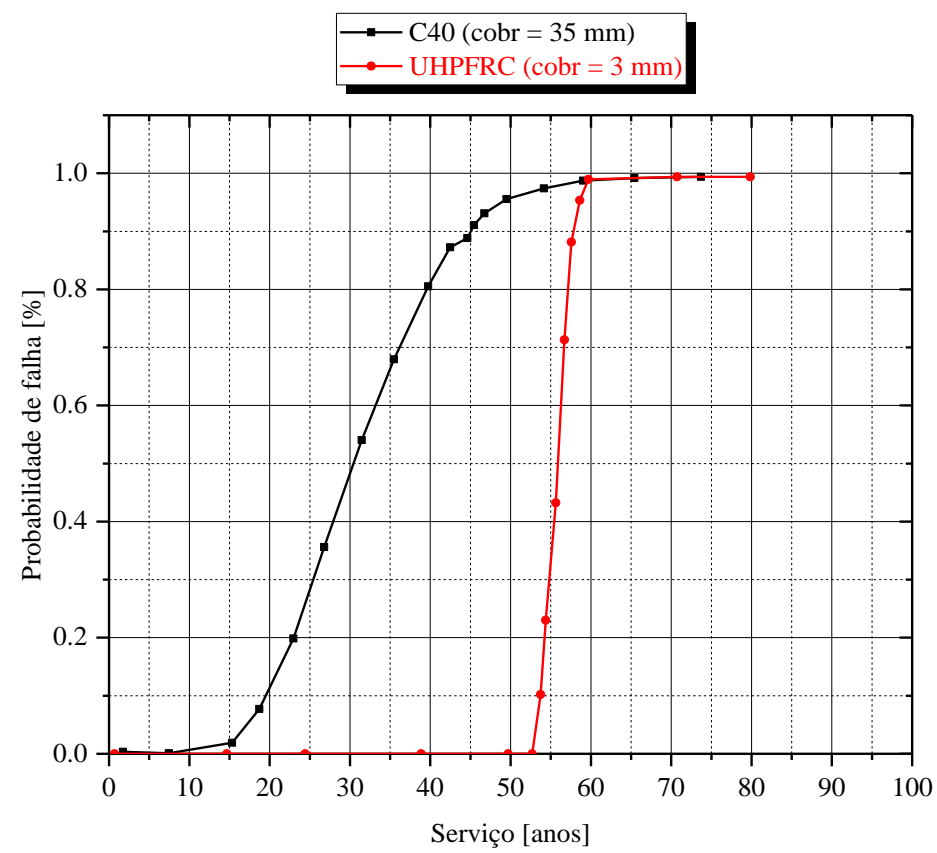

Fonte: Adaptado de Toledo Filho et al. (2012)

\section{APLICAÇÕES EM ESTRUTURAS}

Dada a descrição teórica sobre as características mecânicas e microestruturais do UHPFRC, faz-se necessária a abordagem sobre o emprego do material na indústria da construção civil. Sua primeira aplicação foi em 1997, sendo utilizado na substituição das vigas das torres de refrigeração das usinas nucleares de Cattenom e Civaux, na França (AHLBORN; PUESE; MISSON, 2008; RESPLENDINO, 2004). Ainda em 1997, a ponte Sherbrooke Bridge (fig. 7) foi construída no Canadá, sendo a primeira a utilizar o material (FEHLING et al., 2014).

Atualmente nota-se a tendência de utilização do UHPC/UHPFRC na indústria de pré moldados pois permite algumas vantagens, tais como: reduções da seção transversal, aumento de velocidade de produção, facilidade de manuseio e transporte, além de fases de construção mais rápidas com impacto ambiental reduzido (GRAYBEAL; HARTMANN, 2005). 
No entanto, certos aspectos técnicos explicam o uso limitado em estruturas e desafio tecnológico da aplicação do material, tais como: o alto preço do material, produção em pequena escala, sensibilidade do material ao procedimento de mistura e proporções, variabilidade das propriedades mecânicas em função das condições de moldagem (SPASOJEVIĆ, 2008). Todavia, mesmo dadas as limitações apresentadas, a aplicação do UHPFRC é verificada em: (i) elementos de cobertura (fig. 8), (ii) pontes rodoviárias (fig. 9a, 9b e 10), (iii) passarelas (fig. 11a, 11b, 11c, 12a e 12b), (iv) pilares (fig. 13), (v) tabuleiro de pontes (fig. 14a e 14b) e (vi) elementos de proteção à infraestrutura (FEHLING et al., 2014; SALEEM, 2011; SPASOJEVIĆ, 2008).

Figura 7 - Sherbrooke Bridge

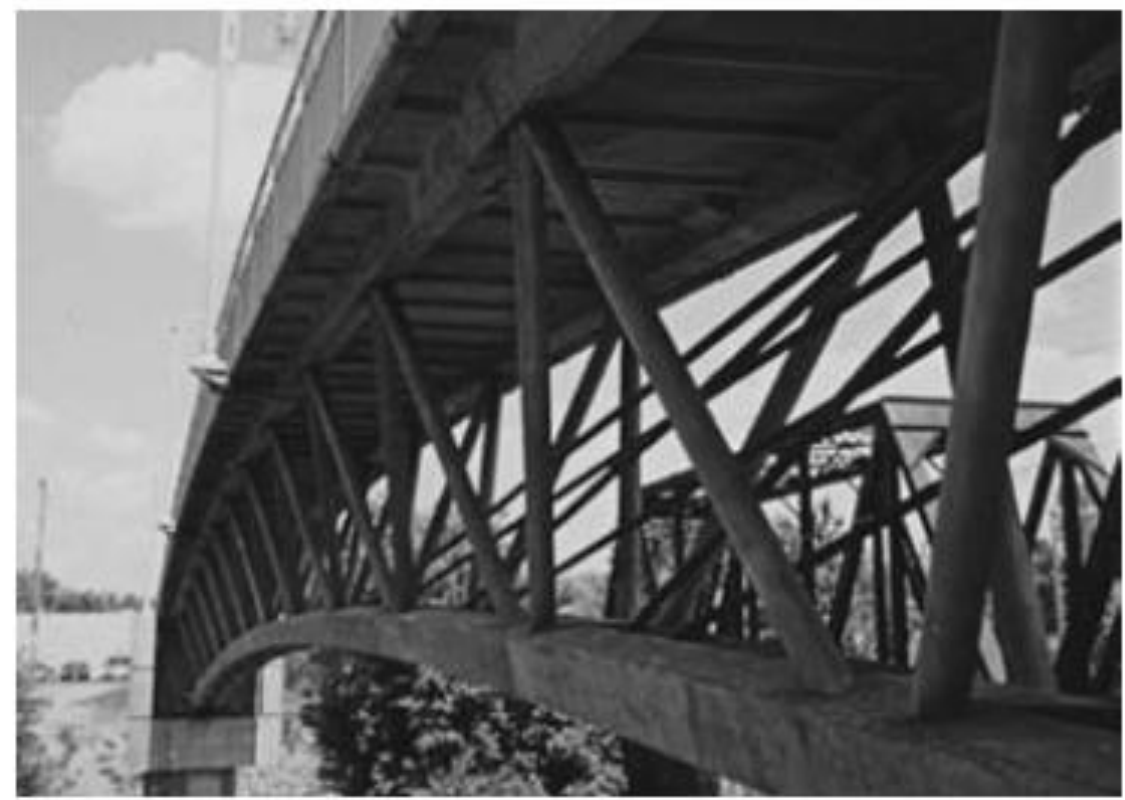

Fonte: Fehling et al. (2014)

Figura 8 - Cobertura do Jean Bouin Stadium, Paris

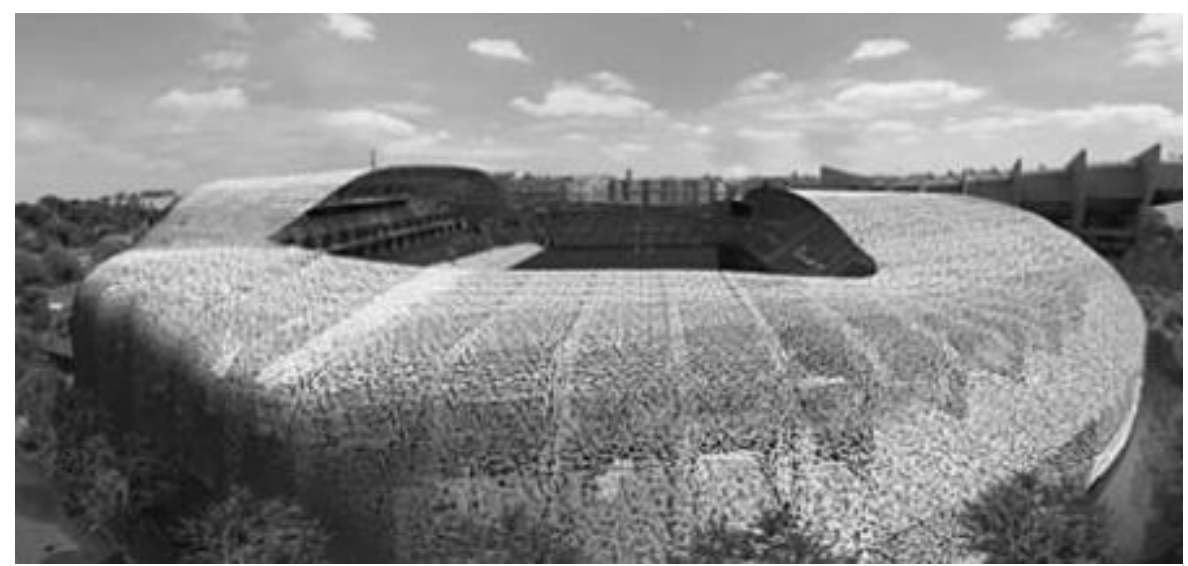

Fonte: Fehling et al. (2014) 
Figura 9 (a) - Corte Transversal Ponte Bourg-lès-Valence

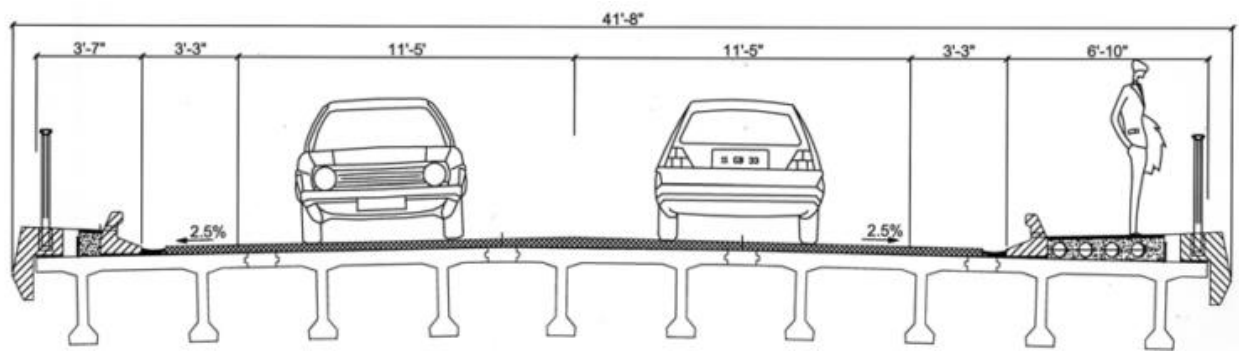

Fonte: Rebentrost e Wight (2008)

Figura 9 (b) - Parte inferior Ponte Bourg-lès-Valence

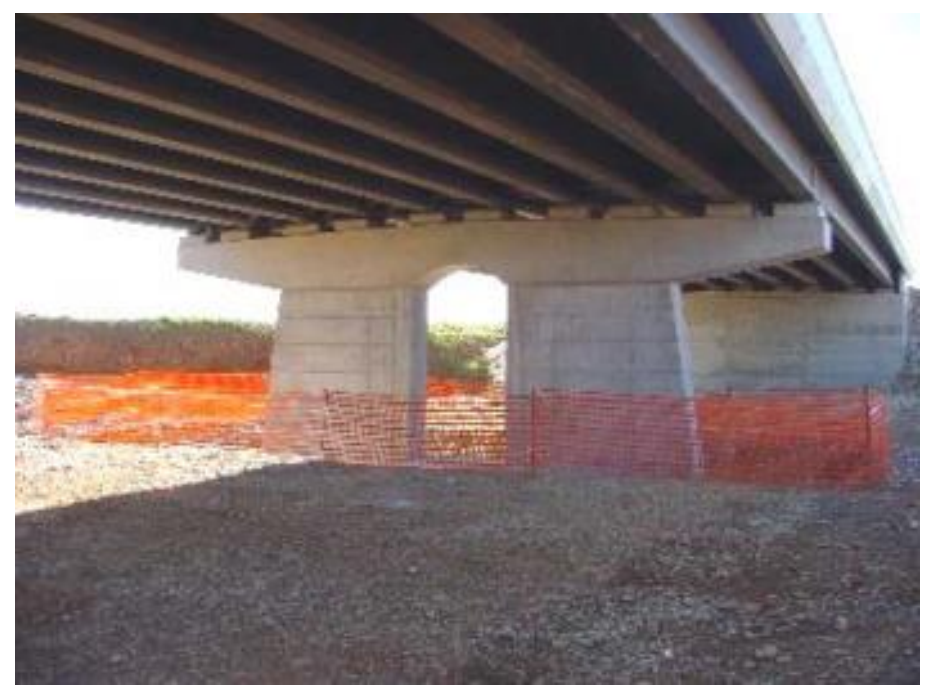

Fonte: Rebentrost e Wight (2008)

Figura 10 - Ponte Shepherds Creek Road, Austrália

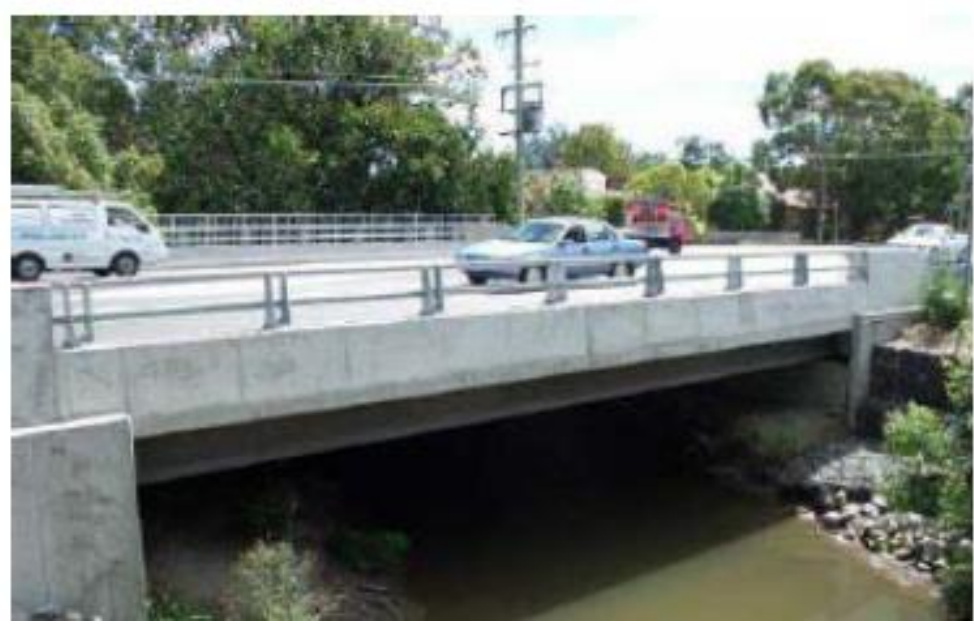

Fonte: Rebentrost e Wight (2008) 
Figura 11 (a) - Elevação típica da seção pi, Papatoetoe and Penrose, Nova Zelândia

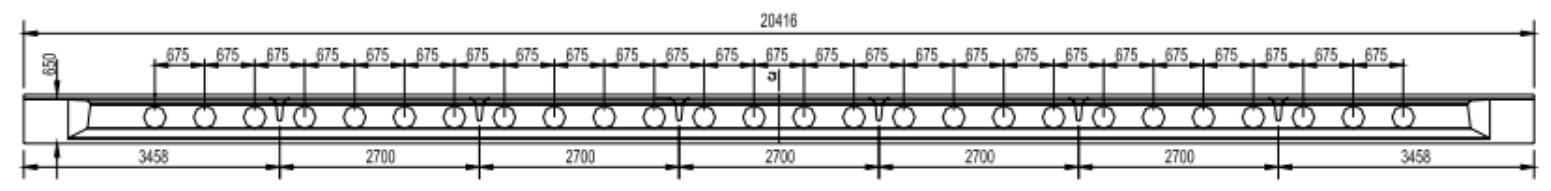

Fonte: Rebentrost e Wight (2008)

Figura 11 (b) - Seção pi, Papatoetoe and Penrose, Nova Zelândia

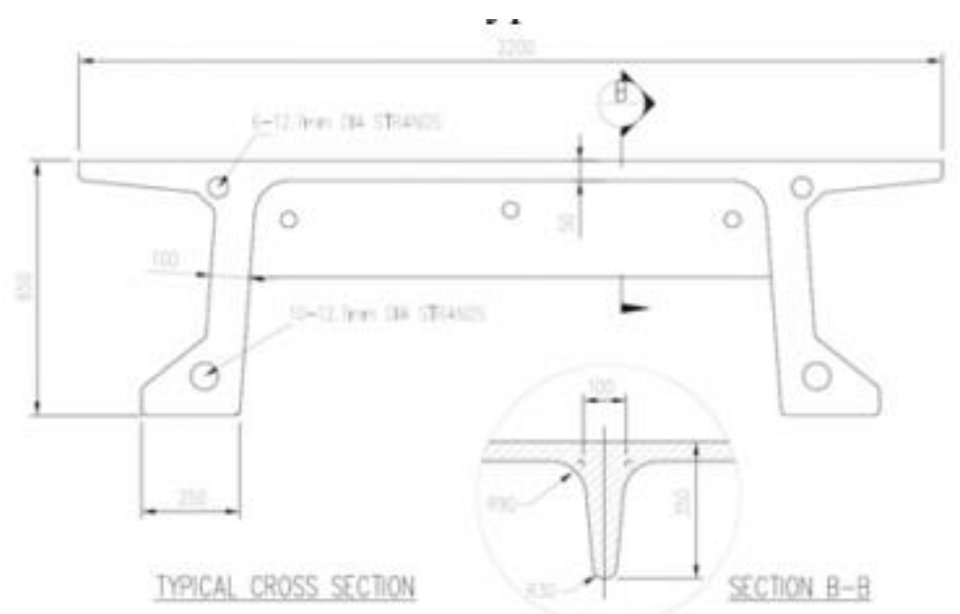

Fonte: Rebentrost e Wight (2008)

Figura 11 (c) - Içamento, Papatoetoe and Penrose, Nova Zelândia

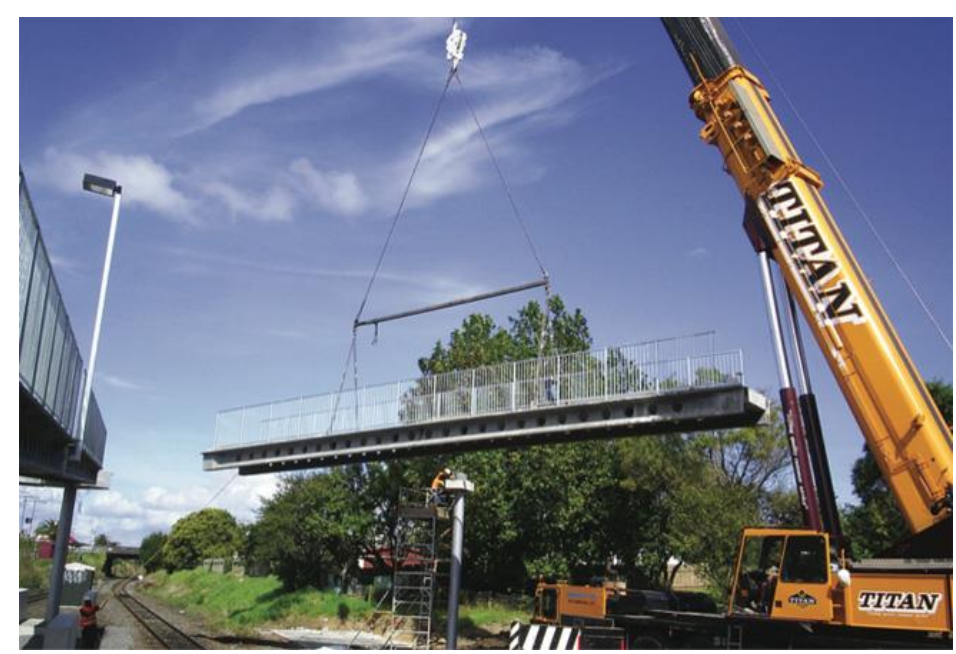

Fonte: Rebentrost e Wight (2008) 
Figura 12 (a) - Vista da seção transversal da passarela de Sakata-Mirai, Japão
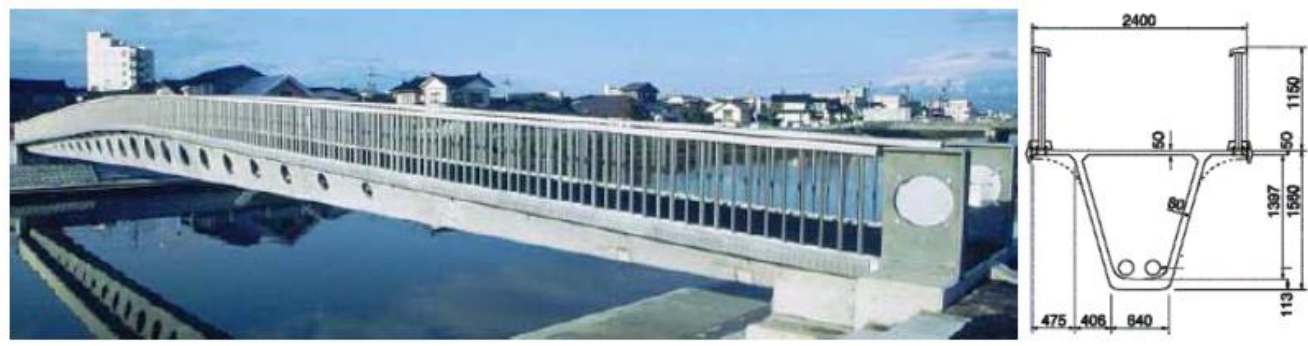

Fonte: Fehling et al. (2014); Rebentrost e Wight (2008)

Figura 12 (b) - Unidade pré-moldada da passarela de Sakata-Mirai, Japão

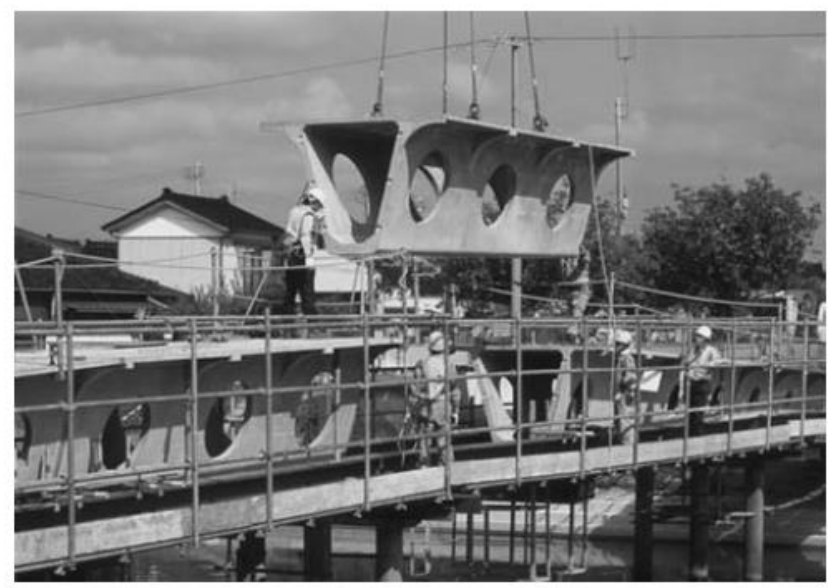

Fonte: Fehling et al. (2014); Rebentrost e Wight (2008)

Figura 13 - Pilares do MuCEM, Marseille

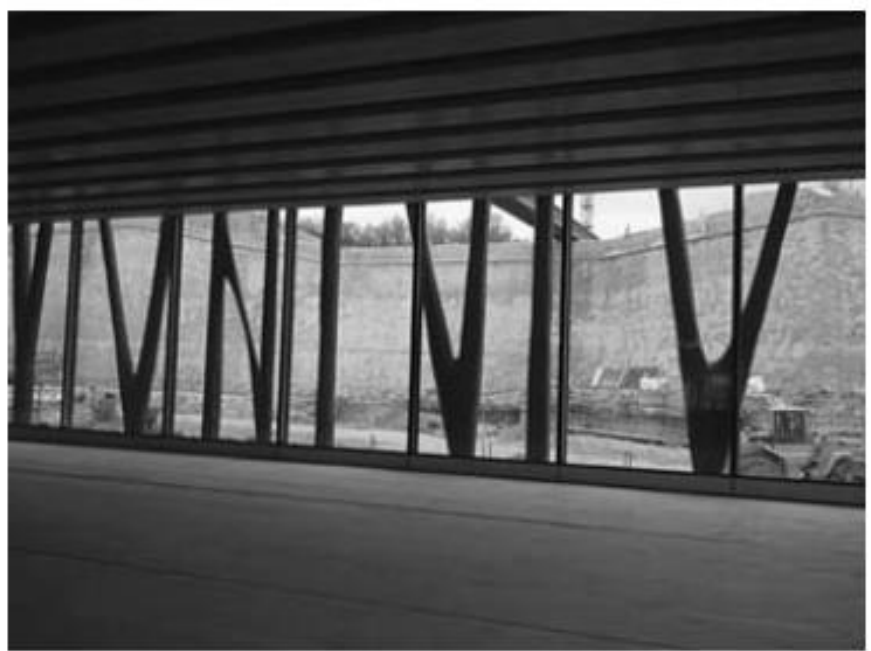

Fonte: Fehling et al. (2014) 
Considerando especialmente a aplicação em elementos pré moldados de lajes e/ou tabuleiros rodoferroviários, destacam-se os trabalhos de (SALEEM, 2011), (SALEEM et al., 2013), (GHASEMI et al., 2016), (VENANCIO; MYERS, 2016), (VENANCIO, 2016), (LI; WU; HAO, 2015) e (MENG; KHAYAT, 2016). (SALEEM, 2011) e (SALEEM et al., 2013) desenvolveram um novo sistema de tabuleiro para pontes móveis utilizando UHPC reforçado por fibras e barras de aço de alta resistência (UHPFRC-HSS). O tabuleiro apresenta forma de "waffle", com vigas primarias e secundárias, conforme as Figuras 14a e 14b. A viabilidade do sistema foi demonstrada por meio de uma série de experimentos com feixes de vigas simples e múltiplas. Ao final do trabalho os autores afirmam que ainda que o peso dos painéis fosse razoavelmente baixo (i.e. $1,55 \mathrm{kN} / \mathrm{m}^{2}$ ) não era suficiente para a aplicação de pontes móveis. Posteriormente, (GHASEMI et al., 2016) melhoraram o mesmo sistema reduzindo o peso próprio para valores inferiores a $1,0 \mathrm{kN} / \mathrm{m}^{2}$ gerando a viabilidade da aplicação.

Figura 14 (a) - Vista inferior de Tabuleiro de ponte móvel

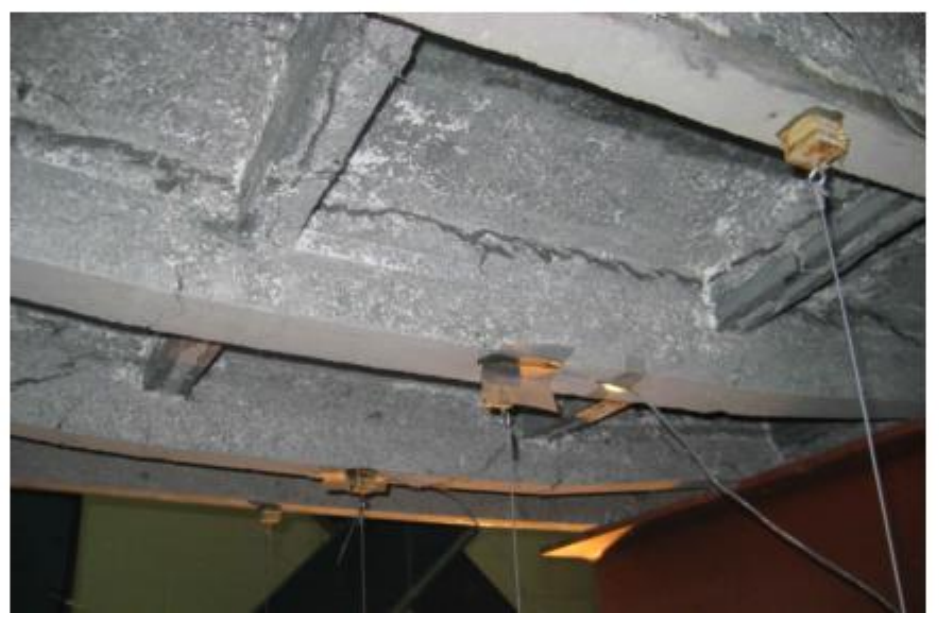

Fonte: Ghasemi et al. (2016); Saleem (2011) 
Figura 14 (b) - Esquema 3D de Tabuleiro de ponte móvel

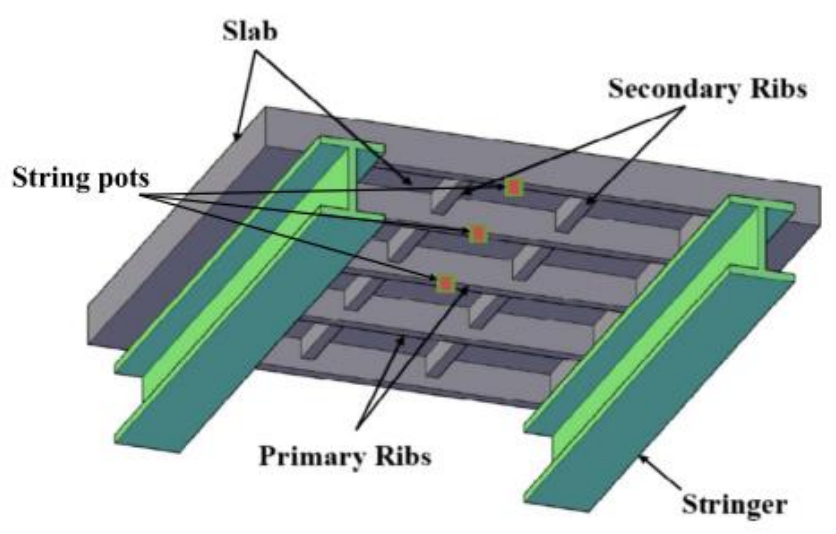

Fonte: Ghasemi et al. (2016); Saleem (2011)

Ainda no campo de aplicação em lajes pré moldadas, (VENANCIO; MYERS, 2016) e (VENANCIO, 2016) comparam o desempenho de painéis maciços sem estribos de UHPFRC em relação ao concreto convencional. No estudo, demonstrou-se grande melhoria quanto à resistência a força cortante dos elementos de concreto de ultra alto desempenho. Investigando a vocação das lajes de UHPFRC para edificações militares, (LI; WU; HAO, 2015) avaliam por meio de ensaios com cargas explosivas (i.e. massa de TNT variando entre 1,0 - 14,0 kg) a superioridade do UHPFRC em comparação aos concretos convencionais. (MENG; KHAYAT, 2016) propõem a substituição do concreto convencional por UHPFRC em dormentes de linhas ferroviárias de alta velocidade. Infere-se que a utilização de meia espessura de UHPFRC nos dormentes gera o aumento em respectivamente $185 \%, 28 \%$ e $412 \%$ do carregamento máximo, rigidez e energia dissipada na ruptura do elemento.

Em suma, nota-se que o UHPFRC é um material emergente na indústria da construção civil. Seu grande potencial é notável para várias aplicações, muitas das quais ainda não reconhecidas, fornecendo soluções econômicas, sustentáveis e inovadoras em áreas em que o concreto normal luta para encontrar uma solução plausível. Entretanto, devido as limitações apresentadas é necessário superar os desafios tecnológicos para sua aplicação, contribuindo para que a produção em grande escala de elementos de UHPC/UHPFRC cresça continuamente. 


\section{CONSIDERAÇÕES FINAIS}

O presente trabalho apresenta resultados sobre as propriedades mecânicas do UHPFRC. Fica evidente que o UHPFRC é um material emergente, com propriedades superiores aos concretos convencionais e de alta resistência, podendo ser usado com sucesso em situações onde os concretos usuais não são competitivos.

\section{REFERÊNCIAS}

AFGC (ASSOCIATION FRANCAISE DE GÉNIE CIVIL). Ultra high performance fibre-reinforced concretes, recommendations. 2013.

AHLBORN, T. M.; PUESE, E. J.; MISSON, D. L. Ultra-high performance concrete for Michigan bridges, material performance: phase I. Michigan. Dept. of Transportation, 2008.

AÏTCIN, P. C. Binders for durable and sustainable concrete. 1a ed., Londres: CRC Press. Taylor \& Francis, 2007.

ALI, A. Behaviour of Prestressed Ultra-High PerformanceConcrete I-Beams Subjected to Shear and Flexure. Dissertation (Master in Applied Sciences in Civil Engineering) - University of Ottawa, Canada, 2013.

BALESTRA, C. E. T.; OZELAME, J. S.; SAVARIS, G. Efeito da adição de fibras de aço e poliméricas na resistência à compressão do concreto de ultra alto desempenho. REEC - Revista Eletrônica de Engenharia Civil, v. 16, n. 1, p. 26-35, 2020 .

BOULEKBACHE, B.; HAMRAT, M.; CHEMROUK, M.; AMZIANE, S. Flowability of fibre-reinforced concrete and its effect on the mechanical properties of the material. Construction and Building Materials. V. 24, n. 9, p. 1664-1671, 2010.

BUTTIGNOL, T.E.T.; SOUSA, J.L.A.O.; BITTENCOURT, T.N. Ultra Concreto Reforçado com Fibra de Alto Desempenho (UHPFRC): uma revisão das propriedades dos materiais e procedimentos de projeto. Revista IBRACON de Estruturas e Materiais. v. 10, n. 4, p. 957-971, 2017.

CAVILL, B.; REBENTROST, M.; PERRY, V. Ductal-a high-performance material for resistance to blasts and impacts. Australian Journal of Structural Engineering, v. 7, n. 1, p. 37-45, 2006.

CHEYREZY; RICHARD, P. Reactive powder concretes with high ductility and 200 - 800 mpa compressive strength. Special Publication ACI, v. 144, 1994. 
CORINALDESI, V.; MORICONI, G. Mechanical and thermal evaluation of ultra high performance fiber reinforced concretes for engineering applications.

Construction and Building Materials, v. 26, n. 1, p. 289-294, 2012.

DE LARRARD, F.; SEDRAN, T. Optimization of ultra-high-performance concrete by the use of a packing model. Cement and Concrete Research, v. 24, n. 6, p. 9971009, 1994.

FEHLING, E. et al. Ultra-high performance concrete UHPC. Berlin: Ernst \& Sohn, 2014.

GHASEMI, S. et al. A super lightweight UHPC-HSS deck panel for movable bridges. Journal: Engineering Structures, v. 113, p. 186-193, 2016.

GRAYBEAL, B.; TANESI, J. A Cementitious Long-Life Wearing Course to Reduce Frequency of Maintenance Works on High-Traffic Roads. Transport Research Arena Europe 2008. Anais [...] Ljubljana: 2008.

GRAYBEAL, A. A.; HARTMANN, J. L. Construction of an Optimized UHPC Vechicle Bridge. ACI Special Publication, v. 228, 2005.

GUZZO, G. Avaliação do comportamento do concreto convencional e do concreto de ultra-alto desempenho frente à contaminação por cloretos. 2018. Graduação (Trabalho de Conclusão de Curso) - Universidade Tecnológica Federal do Paraná, Curitiba, 2018.

HABEL, K. et al. Development of the mechanical properties of an ultra-high performance fiber reinforced concrete (UHPFRC). Cement and Concrete Research, v. 36, n. 7, p. 1362-1370, 2006.

HABEL, K.; GAUVREAU, P. Response of ultra-high performance fiber reinforced concrete (UHPFRC) to impact and static loading. Cement and Concrete Composites, v. 30, n. 10, p. 938-946, 2008.

HANNA, K.; MORCOUS, G.; TADROS, M. Effect of supplementary cementitious materials on the performance of concrete pavement. Journal of Materials in Civil Engineering, v. 26, n.4, p. 789-793, 2014.

HANNAWI, K.; BIAN, H.; PRINCE-AGBODJAN, W.; RAGHAVAN, B. Effect of different types of fibers on the microstructure and the mechanical behavior of Ultra-High Performance Fiber-Reinforced Concretes. Composites Part B:

Engineering, v. 86, p. 214-220, 2016.

HUNG, C.-C.; LEE, H.-S.; CHAN, S. N. Tension-stiffening effect in steel-reinforced UHPC composites: constitutive model and effects of steel fibers, loading patterns, and rebar sizes. Composites Part B: Engineering, v. 158, p. 269-278, 2019.

LI, J.; WU, C.; HAO, H. An experimental and numerical study of reinforced ultrahigh performance concrete slabs under blast loads. Materials \& Design, v. 82, p. 6476, 2015.

MÁCA, P.; SOVJÁK, R.; VAVŘINÍK, T. Experimental Investigation of Mechanical Properties of UHPFRC. Procedia Engineering, v. 65, p. 14-19, 2013. 
MENG, W.; KHAYAT. Flextural performance of ultra-high performance concrete ballastless track slabs Weina. In: INTERNATIONAL CONFERENCE ON UHPC MATERIALS AND STRUCTURES, 1, p.1-7, Anais [...] Columbia: 2016.

MOHAMMED, B. H.; SHERWANI, A. F. H.; FARAJ, R. H.; QADIR, H. H.; YOUNIS, K. H.; Mechanical properties and ductility behavior of ultra-high performance fiber reinforced concretes: Effect of low water-to-binder ratios and micro glass fibers. Ain Shams Engineering Journal, v. 12, n. 2, p. 1557-1567, 2020.

PENG, Y.; HU, S.; DING, Q. Preparation of reactive powder concrete using fly ash and steel slag powder. Journal Wuhan University of Technology. Materials Science Edition, v. 25, n. 2, p. 349-354, 2010.

PORTENEUVE, C.; KORB, J.; PETIT, D. Nuclear magnetic resonance characterization of high- and ultrahigh-performance concrete Application to the study of water leaching. Cement and Concrete Research, v. 31, p. 1887-1893, 2001.

REBENTROST, M.; WIGHT, G. Experience and applications of ultra high performance concrete in Asia. In: INTERNATIONAL SYMPOSIUM ON ULTRA HIGH PERFORMANCE CONCRETE, 2, 2008. Proceedings [...] 2008.

RESPLENDINO, J. First recommendations for Ultra-High-Performance Concretes and examples of application. Proceedings of the International Symposium on Ultra High Performance Concrete. Anais [...] Kassel, 2004.

RICHARD, P.; CHEYREZY, M. Composition of reactive powder concretes. Cement and Concrete Research, v. 25, n. 7, p. 1501-1511, 1995.

ROMPA, D. D.; GIDRÃO, G. de M. S. Caracterização mecânica da rigidez e resistência do concreto de ultra-alto desempenho reforçado por fibras (UHPFRC). Ambiente Construído, Porto Alegre, v. 20, n. 2, p. 73-88, 2020.

RUSSEL, G. H.; GRAYBEAL, B. A. Ultra-high performance concrete: a state-ofthe-art report for the bridge community. Federal Highway Administration of United States Office of Infrastructure Research and Development, 2013.

SALEEM, M. A. Alternatives to steel grid bridge decks. 2011. Dissertation (Tesis in Civil Engineering) - Florida International University, Florida, 2011.

SALEEM, M. A. et al. Development length of high-strength steel rebar in ultrahigh performance concrete. Journal of Materials in Civil Engineering, v. 25, n. 8, p. 991998, 2013.

SANCHEZ, F.; SOBOLEV, K. Nanotechnology in concrete - A review. Construction and Building Materials, v. 24, n. 11, p. 2060-2071, 2010.

SHAIKH, F. U. A.; LUHAR, S.; AREL, H. S.; LUHAR, I. Performance evaluation of Ultrahigh performance fibre reinforced concrete $-\mathbf{A}$ review. Construction and Building Materials, v. 232, 2020.

SHI, C. et al. The hydration and microstructure of ultra high-strength concrete with cement-silica fume-slag binder. Cement and Concrete Composites, v. 61, p. 44$52,2015$. 
SHI, Y.; MATSUI, I.; FENG, N. Effect of compound mineral powders on workability and rheological property of HPC. Cement and Concrete Research, v. 32, n. 1, p. 71-78, 2002.

SPASOJEVIĆ, A. Structural implications of ultra-high performance fibrereinforced concrete in bridge design. Epfl, v. 4051, p. 203, 2008.

TAFRAOUI, A. et al. Metakaolin in the formulation of UHPC. Construction and Building Materials, v. 23, n. 2, p. 669-674, 2009.

TOLEDO FILHO, R. D. et al. Performance assessment of ultra high performance fiber reinforced cementitious composites in view of sustainability. Materials and Design, v. 36, p. 880-888, 2012.

TORREGROSA, E. C. Dosage optimization and bolted connections for UHPFRC ties. 2013. Ph.D Thesis (Doctor of Sciences UPV) - Universitat Politècnica de València, Spain, 2013.

TUAN, N. van et al. Hydration and microstructure of ultra high performance concrete incorporating rice husk ash. Cement and Concrete Research, v. 41, n. 11, p. 1104-1111, 2011.

VAN TUAN, N. et al. The study of using rice husk ash to produce ultra high performance concrete. Construction and Building Materials, v. 25, n. 4, p. 2030-2035, 2011.

VENANCIO, V. G. Behavior of Ultra-High Performance Concrete Bridge Deck Panels Compared to Conventional Stay-in-Place Deck Panels. 2016. Thesis (Master theses Architectural and Environmental Engineering civil) - Missouri University Of Science And Technology, U.S.A., 2016.

VENANCIO, V. G.; MYERS. Behavior of Ultra-High Performance Concrete Bridge Deck Panels Compared to Conventional Stay-in-Place Deck Panels. First International Interactive Symposium on UHPC. Anais [...] 2016.

WANG, C. et al. Preparation of Ultra-High Performance Concrete with common technology and materials. Cement and Concrete Composites, v. 34, n. 4, p. 538-544, 2012.

WANG, D. et al. A review on ultra high performance concrete: part II: hydration, microstructure and properties. Construction \& Building Materials, v. 96, p. 368-377, 2015 .

WILLE, K. et al. Ultra-high performance concrete and fiber reinforced concrete: achieving strength and ductility without heat curing. Materials and Structures, v. 45, n. 3, p. 309-324, 2011.

WU, Z. et al. Effects of steel fiber content and shape on mechanical properties of ultra high performance concrete. Construction \& Building Materials, v. 103, p. 8-14, 2016.

YAN, J. et al. Ultimate strength behaviour of S-UHPC-S and SCS sandwich beams under shear loads. Journal of Constructional Steel Research, v. 149, p.195-206, 2018. 
YAZICI, H. et al. Mechanical properties of reactive powder concrete for different curing regimes. International Journal of Earth Sciences and Engineering, v. 8, n. 6, p. 2698-2702, 2015.

YAZICI, H. et al. Utilization of fly ash and ground granulated blast furnace slag as an alternative silica source in reactive powder concrete. Fuel, v. 87, n. 12, p. 24012407, 2008.

YOO, D.-Y.; LEE, J.-H. ; YOON, Y.-S. Effect of fiber content on mechanical and fracture properties of ultra high performance fiber reinforced cementitious composites. Composite Structures, v. 106, p. 742-753, 2013.

YU, R.; P. SPIESZ, P; BROUWERS, H.J.H. Development of an eco-friendly UltraHigh Performance Concrete (UHPC) with efficient cement and mineral admixtures uses. Cement and Concrete Composites, v. 55, p.383-394, 2015.

YU, R.; SPIESZ, P.; BROUWERS, H. J. H. Mix design and properties assessment of Ultra-High performance Fibre Reinforced Concrete (UHPFRC). Cement and Concrete Research, v. 56, p. 29-39, 2014.

ZDEB, T. Ultra-high performance concrete: properties and technology. Bulletin of the Polish Academy of Sciences, v. 61, n. 1, p. 183-193, 2013.

ZHANG, Peng; LI, Qing-Fu. Durabilidade de compósitos de concreto de alto desempenho contendo sílica ativa. Proceedings of the Institution of Mechanical Engineers, Part L: Journal of Materials: Design and Applications, v. 227, n. 4, p. 343349, 2013.

ZHU, W.; GIBBS, J. C. Use of different limestone and chalk powders in selfcompacting concrete. Cement and Concrete Research, v. 35, n. 8, p. 1457-1462, 2005. 